\title{
Electromagnetic field quantization in planar absorbing heterostructures
}

\author{
V.I. Pipa \\ V. Lashkaryov Institute of Semiconductor Physics, NAS of Ukraine, \\ 41, prospect Nauky,03028Kyiv, Ukraine; e-mail: pipa1@bigmir.net
}

\begin{abstract}
The quantization scheme for the electromagnetic field in planar absorbing heterostructures has been developed. The scheme is based on the field expansion over a complete set of orthonormal modes. We used two types of the field modes. The first one is defined as the field created by a plane wave incident at the surface of the structure from the non-absorbing half space. The second type of modes corresponds to the field generated by electric current fluctuations in the absorbing media. To normalize the field modes, the following conditions were used: 1) the time-averaged Poynting vector attributed to the incident wave equals the density of energy flow of elementary quanta of the field energy; 2) for the given frequency and polarization, the total time-averaged Poynting vector equals to zero. The theory is applied to calculate the rate of spontaneous transitions between electron subbands in a quantum well placed near the absorbing layer that can support the surface phonon or plasmon polaritons.
\end{abstract}

Keywords: electromagnetic field, quantization, absorbing heterostructure, spontaneous emission.

Manuscript received 16.09.10; accepted for publication 02.12.10; published online 28.02.11.

\section{Introduction}

In the conventional quantization scheme, the electromagnetic field is expressed in terms of a set of orthonormal modes; each mode is quantized as a harmonic oscillator. For free space, the plane-wave modes that are defined using the periodical boundary conditions in a fiction quantization box are usually used. The field quantization in the presence of bounded nonabsorbing media has been carried out for plane-parallel layers [1, 2] and semi-infinite dielectrics [3, 4]. For such systems, the eigenmodes of Maxwell's equations with the appropriate boundary conditions are used.

The methods of quantization developed for nonabsorbing media fail when losses are present [5]. The quantization problem in absorbing media has been considered by many authors, and many different schemes have been proposed (see, e. g., [6] for a review). In this paper, we have modified the approach [7-11] based on the introduction of noise currents into the Maxwell equations for the macroscopic electromagnetic field. In the works [7-11], the current correlation function has been postulated but not derived. This function is taken in the form that ensures preservation of the known canonical field commutation relations in the presence of absorption. Here, for the electromagnetic field in the presence of absorbing and dispersing planar structures, we develop the quantization scheme based on the mode decomposition. In order to calculate the field generated by some absorbing medium, the oscillator model is employed. The modes are normalized with respect to the electromagnetic energy flow. It is assumed that the total normal electromagnetic energy flow through an arbitrary plane parallel to the interfaces is absent. The theory is applied to calculate the rate of spontaneous photon emission under transitions between electron subbands in a quantum well placed in the near field of an absorbing layer that supports the surface-phonon polaritons or the surface-plasmon polaritons.

\section{Photons in the presence of bounded non-adsorbing dielectrics}

Let us first consider a structure consisting of two nonabsorbing dielectrics with a common planar interface at $z=0$ that spreads infinitely in $x$ and $y$ directions. The optical properties of non-magnetic media $1(z<0)$ and 
$2(z>0)$ are described by the constant real dielectric permittivities $\varepsilon_{1}$ and $\varepsilon_{2}$, respectively. We set the scalar potential equal to zero. For the vector potential $\mathbf{A}$, we use the Coulomb gauge $\nabla \mathbf{A}=0$. We imply periodic boundary conditions in the $x-y$ plane and seek for the solution in the complex-valued form,

$\mathbf{A}(\mathbf{r}, t)=\mathbf{A}(z) e^{i(\mathbf{q r}-\omega t)}$,

where $\mathbf{q}=\left(q_{x}, q_{y}, 0\right)$ is the real propagation vector, and $\omega>0$ is the frequency. The function $\mathbf{A}(z)$ is found from the equation

$\frac{d^{2} \mathbf{A}}{d z^{2}}+\left(\varepsilon(z) k_{0}^{2}-q^{2}\right) \mathbf{A}=0$,

where $\varepsilon(z)=\varepsilon_{1}+\left(\varepsilon_{2}-\varepsilon_{1}\right) \Theta(z), \Theta(z)$ is the Heaviside step-function, $k_{0}=\omega / c$. The electric field $\mathbf{E}$ and magnetic field $\mathbf{H}$ are found from a vector potential: $\mathbf{E}=i k_{0} \mathbf{A}, \quad \mathbf{H}=\nabla \times \mathbf{A}$. At the interface $z=0$, the tangential components of $\mathbf{E}$ and $\mathbf{H}$ are continuous.

The set of modes consists of the fields created by the plane waves that are incident at the interface $z=0$ from either half space $[3,4]$. The modes are specified by the complex quantum number $\mu=\{\omega, \mathbf{q}, v, \varsigma\}$ where $v=s, p$ labels polarization of the incident wave and $\varsigma= \pm$. The subscripts + and - denote propagation of the incident wave to the right and left, respectively. Let $\mathbf{A}_{i v}^{(+)}=A_{i v}^{(+)} \mathbf{e}_{1 v}^{(+)}$and $\mathbf{A}_{i v}^{(-)}=A_{i v}^{(-)} \mathbf{e}_{2 v}^{(-)}$be the amplitudes of the incident waves. The unit vectors of polarization are defined as

$\mathbf{e}_{j s}^{( \pm)} \equiv \mathbf{e}_{s}=\frac{1}{q}\left(q_{y},-q_{x}, 0\right)$,

$\mathbf{e}_{j p}^{( \pm)}=\frac{1}{q k_{0} \sqrt{\varepsilon_{j}}}\left( \pm k_{j z} \mathbf{q},-q^{2}\right)$,

where $k_{j z}=\sqrt{\varepsilon_{j} k_{0}^{2}-q^{2}}$, the subscript $j=1,2$ denotes the media. The mode $\{\omega, \mathbf{q}, v,+\}$ are given by

$\mathbf{A}_{v}^{(+)}(z)=A_{i v}^{(+)}\left(\mathbf{e}_{1 v}^{(+)} e^{i k_{1 z^{z}}}+r_{v 12} \mathbf{e}_{1 v}^{(-)} e^{-i k_{1 z^{z}}}\right), z \leq 0$, $=A_{i v}^{(+)} t_{v 12} \mathbf{e}_{2 v}^{(+)} e^{i k_{2 z} z}, z \geq 0$.

Here, $r_{v j j^{\prime}}$ and $t_{v j j^{\prime}}\left(j \neq j^{\prime}\right)$ are the Fresnel amplitudes of reflection and transmission,

$r_{\mathrm{v} j j^{\prime}}=\frac{k_{j z} \varepsilon_{j^{\prime}}-k_{j^{\prime} z} \varepsilon_{j}}{k_{j z} \varepsilon_{j^{\prime}}+k_{j^{\prime} z} \varepsilon_{j}}, \quad t_{\mathrm{v} j j^{\prime}}=\sqrt{\frac{\varepsilon_{j}}{\varepsilon_{j^{\prime}}}}\left(1+r_{v j j^{\prime}}\right)$.

Let us now calculate the spectral and angular density of energy flow associated with the above introduced modes. To calculate the Poynting vector,

$\mathbf{S}=\frac{c}{4 \pi}[\mathbf{E} \times \mathbf{H}]$ we express the physical fields $\mathbf{E}$ and $\mathbf{H}$ via the real vector potential. For $z$-component of the Poynting vector averaged over time, which corresponds to rightwardspropagating incident wave, we find

$S_{i v z}^{(+)}(\omega, \mathbf{q})=\frac{\omega}{2 \pi}\left|\mathbf{A}_{i v}^{(+)}\right|^{2} k_{1 z}$.

Replacing $k_{1 z}$ with $-k_{2 z}$ and $\mathbf{A}_{i v}^{(+)}$with $\mathbf{A}_{i v}^{(-)}$in Eq. (7), we get the classical expression for the density of the normal flow $S_{i v z}^{(-)}$associated with the leftwardspropagating incident wave. The same values associated with a photon with the energy $\hbar \omega$ are given by

$S_{i v z}^{(+)}=-S_{i v z}^{(-)}=\frac{\hbar \omega}{2 \pi L^{2}}$,

where $L^{2}$ is the area of normalization in the $x-y$ plane. Comparing the classical and quantum expressions for the energy flows, we find

$\left|A_{i v}^{(+)}\right|^{2}=\frac{\hbar}{L^{2} k_{1 z}},\left|A_{i v}^{(-)}\right|^{2}=\frac{\hbar}{L^{2} k_{2 z}}$.

In the conventional quantization scheme, the amplitudes $A_{i v}^{( \pm)}$are determined from the orthonormalization condition [3] for scalar product corresponding to the electromagnetic energy density. It is convenient to write this condition in the following form

$\int_{-\infty}^{\infty} d z \mathbf{A}_{\omega v}^{(\varsigma)}(z) \mathbf{A}_{\omega^{\prime} v^{\prime}}^{*\left(\varsigma^{\prime}\right)}(z) \varepsilon(z)=\frac{2 \pi \hbar c^{2}}{\omega L^{2}} \delta_{v v^{\prime}} \delta_{\zeta \varsigma^{\prime}} \delta\left(\omega-\omega^{\prime}\right)$

The amplitude factors $\left|A_{i v}^{( \pm)}\right|^{2}$ determined from Eq. (10) with $v, \varsigma=v^{\prime}, \varsigma^{\prime}$ coincide with those given by Eq. (9). Thus, the mode normalizations with respect of the density of electromagnetic energy and the density of energy flow lead to the same results.

For $z \leq 0$, the time-averaged density of energy flows $S_{v z}^{( \pm)}$associated with the modes are given by

$S_{v z}^{(+)}=\frac{\left(1-R_{v 12}\right)}{2 \pi}\left|A_{i v}^{(+)}\right|^{2} \omega k_{1 z}$,

$S_{v z}^{(-)}=-\frac{T_{v 21}}{2 \pi}\left|A_{i v}^{(-)}\right|^{2} \omega k_{1 z}$.

Here, $\quad R_{\mathrm{v} 12}=\left|r_{\mathrm{v} 12}\right|^{2}$ and $T_{\mathrm{v} 21}=\left|t_{\mathrm{v} 21}\right|^{2}$ are the reflection and transmission coefficients. Using Eqs. (5) and (9), we obtain

$S_{v z}^{(-)}+S_{v z}^{(+)}=0$.

Obviously, equality (13) is valid in dielectric 2 as well. Thus, for photon modes of each polarization, the leftwards- and the rightwards-propagating energy flows compensate each other, and the total energy flow through an arbitrary plane parallel to the interface is absent. It is worth to mention that a definition of the 
electromagnetic-energy flow, contrary to that of the electromagnetic energy, remains valid in an absorbing medium as well. In the next Section, we describe the field quantization in the presence of an absorbing medium based on the mode normalization with respect to the density of electromagnetic energy flow.

\section{Photon modes in the presence of absorbing medium}

We now consider the structure consisting of the nonabsorbing dielectric $1(z<0)$ and absorbing dielectric 2 $(z>0)$. The material 2 is characterized by the complex permittivity $\varepsilon_{2}(\omega)=\varepsilon_{2}^{\prime}(\omega)+i \varepsilon_{2}^{\prime \prime}(\omega)$. We set the scalar potential equal to zero. For the one-interface structure, we choose two types of the field modes. The first one formally coincides with the considered above $\{\omega, \mathbf{q}, v,+\}$ modes (Eqs (4), (5) with the complex function $\varepsilon_{2}(\omega)$ ). The second type of modes corresponds to the electromagnetic field generated by the noise-current fluctuations in the absorbing medium 2 . It should be noted that the modes of the first type are defined in the absence of the noise current, and the field generated by the current fluctuations is calculated in the absence of the external incident waves.

To calculate the field emitted by the absorbing medium 2, we divide its volume into small cubic cells of the volume $\Delta V$ and assume that a point electric dipole with the moment $\mathbf{p}(\mathbf{m}) e^{-i \omega t}$ is placed in the center $\mathbf{m}$ of each cell. The vectors $\mathbf{p}(\mathbf{m})$ are assumed to have random orientations; their magnitude $p_{0}$ does not depend on position and orientation. This model corresponds to the isotropic and homogeneous medium. The vector potential $\mathbf{A}_{\mathbf{m}}$ of the field emitted by the oscillator $\mathbf{m}$ satisfies the equation

$$
\Delta \mathbf{A}_{\mathbf{m}}-\nabla\left(\nabla \mathbf{A}_{\mathbf{m}}\right)+k_{0}^{2} \varepsilon(z) \mathbf{A}_{\mathbf{m}}=4 \pi i k_{0} \mathbf{p} \delta(\mathbf{r}-\mathbf{m}) \Theta(z) .
$$

The solution of Eq. (14) obeying the outgoingwave boundary conditions as $z \rightarrow \pm \infty$ has been obtained in [12]. Writing

$$
\mathbf{A}_{\mathbf{m}}(\mathbf{r}, t)=\sum_{\mathbf{q}} \mathbf{A}\left(\mathbf{q}, z, m_{z}\right) e^{i \mathbf{q}(\mathbf{r}-\mathbf{m})-i \omega t}
$$

we have for $z \leq 0$ and $m_{z} \rightarrow z^{\prime}$

$$
\mathbf{A}\left(\mathbf{q}, z, z^{\prime}\right)=\sum_{v} \frac{2 \pi k_{0} t_{v 21}}{k_{2 z}} e^{i k_{2 z^{2}}}\left(\mathbf{e}_{2 v}^{(-)} \mathbf{p}\right) \mathbf{e}_{1 v}^{(-)} e^{-i k_{1 z^{z}}} .
$$

Assuming that the fields emitted by different oscillators are incoherent, we replace the time-averaged Poynting vector by a sum of the partial Poynting vectors corresponding to radiation of separate cells. Therefore, the density of flow emitted to the outside of the absorbing dielectric is given by

$$
S_{z}^{(e m)}=\frac{\omega}{2 \pi} \sum_{\mathbf{m}}\left|\mathbf{A}_{\mathbf{m}}\right|^{2} \operatorname{Re} k_{1 z} .
$$

This expression has to be averaged over all possible directions of the dipole moment $\mathbf{p}$. Let $\langle f(\mathbf{p})\rangle$ denotes the orientation average of the function $f(\mathbf{p})$. Replacing the discreet variable $\mathbf{m}$ by the continuous vector $\mathbf{r}^{\prime}$, we calculate the sum by the integration,

$\sum_{\mathbf{m}} \rightarrow \frac{1}{\Delta V} \int_{0}^{\infty} d z^{\prime} \int_{L^{2}} d x^{\prime} d y^{\prime}$

Upon the integration over $x^{\prime}$ and $y^{\prime}$, the terms with $\mathbf{q}^{\prime} \neq \mathbf{q}$ and $v^{\prime} \neq v$ disappear (in Eq. (17), the averages over the orientations do not depend on $\mathbf{m}$ ). We get

$\left\langle\left|\left(\mathbf{e}_{s} \mathbf{p}\right)\right|^{2}\right\rangle=\frac{\left|p_{0}\right|^{2}}{3},\left\langle\left|\left(\mathbf{e}_{2 p}^{(-)} \mathbf{p}\right)\right|^{2}\right\rangle=\frac{\left|p_{0}\right|^{2}}{3} \frac{\mathbf{q}^{2}+\left|k_{2 z}\right|^{2}}{\left(k_{0} q\right)^{2}\left|\varepsilon_{2}\right|}$

The integration over $z^{\prime}$ yields

$\left\langle S_{z}^{(e m)}\right\rangle=\sum_{v, \mathbf{q}}\left\langle S_{v z}^{(e m)}(\omega, \mathbf{q})\right\rangle$

where

$\left\langle S_{v z}^{(e m)}(\omega, \mathbf{q})\right\rangle=-\frac{2 \pi\left|p_{0}\right|^{2} \omega}{3 L^{2} \Delta V \varepsilon_{2}^{\prime \prime}}\left(1-R_{v 12}\right)$.

To determine the unknown parameter $\left|p_{0}\right|^{2} / \Delta V$, we use the fact that for the modes of each polarization for the leftwards- and the rightwards-propagating energy flows compensate each other. Substituting the result of Eq. (21) into Eq. (13) for $S_{v z}^{(-)}$, we get

$\left|p_{0}\right|^{2}=\frac{3 \hbar \Delta V \varepsilon_{2}^{\prime \prime}}{4 \pi^{2}}$

Let $\mathbf{A}_{0 v}^{(-)} \equiv A_{0 v}^{(-)} \mathbf{e}_{2 v}^{(-)}$be the amplitude of a wave incident at the interface $z=0$ from a volume of the absorbing medium. The transmitted wave $(z \leq 0)$ is given by

$\mathbf{A}_{v}^{(-)}(\mathbf{q}, \mathbf{r}, t)=A_{v}^{(e m)} \mathbf{e}_{1 v}^{(-)} e^{i \mathbf{k}_{1} \mathbf{r}-i \omega t}$,

where $A_{v}^{(e m)} \equiv A_{0 v}^{(-)} t_{v 21}, \mathbf{k}_{1}=\left(\mathbf{q},-k_{1 z}\right)$, and

$A_{0 v}^{(-)}=-\frac{2 \pi i \omega k_{0}}{k_{2 z}} \int_{0}^{\infty} d z^{\prime}\left(\mathbf{e}_{2 v}^{(-)} \mathbf{p}\left(z^{\prime}\right)\right) e^{i k_{2 z^{\prime}} z^{\prime}}$

The orientation-averaged amplitude factors become

$\left\langle\left|A_{0 s}^{(-)}\right|^{2}\right\rangle=\frac{\hbar k_{2 z}^{\prime}}{\left|k_{2 z}\right|^{2} L^{2}}, \quad\left\langle\left|A_{0 p}^{(-)}\right|^{2}\right\rangle=\frac{\hbar k_{2 z}^{\prime}\left(\left|k_{2 z}\right|^{2}+q^{2}\right)}{\varepsilon_{1}\left|\varepsilon_{2}\right|\left(\left|k_{2 z}\right| k_{0} L\right)^{2}}$

$\left\langle\left|A_{v}^{(e m)}\right|^{2}\right\rangle=E_{v}\left|A_{i v}^{(+)}\right|^{2}$

where $E_{v}=1-R_{\mathrm{v} 12}$ is the emissivity of the semi-infinite media 2. We will call the plane waves (23) by $\{\omega, \mathbf{q}, v, e m\}$ modes. In the limit $\varepsilon_{2}^{\prime \prime} \rightarrow 0$, the amplitudes (25) coincide with those given by Eq. (9). In this case, the wave (23) corresponds to $\{\omega, \mathbf{q}, v,-\}$ mode $(z \leq 0)$. 
Thus, in the half space $z \leq 0$, the electromagnetic field (13) can be presented by the modes $\{\omega, \mathbf{q}, v,+\}$ (Eqs (4), (9)) and the modes $\{\omega, \mathbf{q}, v, e m\}$ (Eqs (23), (25), (26)):

$\mathbf{A}(\mathbf{r}, t)=\sum_{\mu} a_{\mu} \mathbf{A}_{\mu}(z) e^{i \mathbf{q r}-i \omega t}+$ c.c.

Here $\Sigma_{\mu}$ denotes the summation over $\mathbf{q}, \quad v$, $\varsigma=+, e m$ and the integration over the frequency $(0 \leq \omega \leq \infty)$. The quantization of the field is to regard the coefficients $a_{\mu}$ and $a_{\mu}^{*}$ in Eq. (27) as a photon annihilation and creation operators, respectively. Note that the appropriate solutions of Eq. (14) for $z \geq 0$, with the magnitude of the dipole moment given by Eq. (22), provide the quantum description of the electromagnetic field inside the absorbing dielectric 2 as well. One can show that the operator-valued $\hat{\mathbf{A}}$ (Eq. (27)) satisfies the commutation relation

$\left[A_{l}(\mathbf{r}, t), \frac{\partial A_{k}\left(\mathbf{r}^{\prime}, t\right)}{\partial t}\right]=4 \pi i c^{2} \hbar \delta_{l k} \delta\left(\mathbf{r}-\mathbf{r}^{\prime}\right)$, [7-11].

which has been postulated but not derived in works

The obtained results may be extended to multilayer absorbing structures. Consider, for example, a nontransparent multilayer structure $(z>0)$ bounded by a non-absorbing dielectric $(z \leq 0)$. In the half space $z \leq 0$, the field can be presented by the same modes $\{\omega, \mathbf{q}, v,+\}$ and $\{\omega, \mathbf{q}, v, e m\}$, but the amplitudes of reflection and transmission in Eq. (4) and the emissivity in Eq. (26) have to be replaced by generalized expressions taking into account all interfaces. For semitransparent absorbing structures $(0 \leq z \leq l)$, the mode expansion (27) for $z \leq 0$ includes also the mode $\{\omega, \mathbf{q}, v,-\}-$ the field created by a wave which is incident at the interface $z=l$ from the non-absorbing half space $z>l$. In this case, the coefficient $E_{v}$ in Eq. (26) is the emissivity of the layer $(0 \leq z \leq l)$.

\section{Radiative lifetime of an excited two-level system}

In this Section, we examine the spontaneous decay of an excited two-level atomic system placed near a plane surface of an absorbing medium. It is known (see, e.g., [13] and references therein) that the spontaneous decay is controlled by the configuration and dielectric properties of atom's macroscopic surrounding. In classical electrodynamics, an emitting atom is described as an oscillating point electric dipole driven by the reflected part of its own radiation field. In quantum theory, modification of the spontaneous emission stems from a dependence of the transition probability on the position of the atom. For non-absorbing dielectrics, when the relative emission rate is defined as a ratio of the emission rate to its bulk value, the classical and quantum theories (in dipole approximation) gave the same result [3]. Here, we compare the analogous results for an atomic system in the presence of an absorbing and dispersing medium.

We first consider a two-level atom located at the position $\mathbf{r}_{0}=\left(0,0,-z_{0}\right)$ in the non-absorbing dielectric $1, z_{0}$ is the distance to the absorbing dielectric $(z \geq 0)$. The interaction of electrons with a weak electromagnetic field is describes by

$H_{\mathrm{int}}=-\frac{i \hbar e}{m c}(\mathbf{A} \nabla)$,

where $e$ and $m$ are the charge and mass of electron. For transitions between the first excited state 2 and the ground state 1 , the spontaneous emission rate $1 / \tau \equiv W_{21}$ ( $\tau$ is the radiative lifetime of the excited state and $W_{21}$ is the transition probability) is determined using the Fermi golden rule:

$W_{21}=\frac{2 \pi}{\hbar} \sum_{\mu}\left|M_{12}^{(\mu)}\right|^{2} \delta\left(\hbar \omega-\left(E_{2}-E_{1}\right)\right)$.

Here, $M_{12}^{(\mu)}$ is the matrix element of the transition, $E_{2}$ and $E_{1}$ are the electron energies. The wavelengths of emitted photons are assumed to be large as compared to the atom size. So, the vector potential $\mathbf{A}(\mathbf{r})$ can be replaced with $\mathbf{A}\left(-\mathbf{r}_{0}\right)$ (dipole approximation). Replacing the summation over $\mathbf{q}$ by the integration, we obtain

$W_{21}(\omega)=\frac{\left(k_{0} L\right)^{2}}{2 \pi \hbar^{2}} \sum_{v, \varsigma} \int\left|\left(\mathbf{A}_{v}^{(\varsigma)}\left(-z_{0}\right) \mathbf{d}_{21}\right)\right|^{2} d \mathbf{q}$,

where $\mathbf{d}_{21}$ is the dipole matrix element, $k_{0}=\omega / c$, and $\omega=\left(E_{2}-E_{1}\right) / \hbar$. Let $\tau_{0}$ denotes the radiative lifetime of the atom placed in a boundless dielectric with the real permittivity $\varepsilon_{1}$. Using the bulk modes $\{\omega, \mathbf{q}, v, \pm\}$ $\left(r_{\mathrm{v} 12}=0, t_{\mathrm{v} 21}=1\right)$, we get

$\frac{1}{\tau_{0}}=\frac{4 k_{0}{ }^{3}\left|\mathbf{d}_{21}\right|^{2} \sqrt{\varepsilon_{1}}}{3 \hbar}$.

This rate coincides with that for emission of conventional bulk photons introduced using the periodic boundary conditions [13]. Substituting the functions given in Eqs (4), (9) and Eqs (23), (26) into Eq. (31) for the photon modes, we obtain the following expression after straightforward calculation:

$\frac{\tau_{0}}{\tau}=1+\frac{3}{8 \sqrt{\varepsilon_{1}}} \int_{0}^{\infty} d \xi \operatorname{Re}\left\{\frac{r}{\sqrt{\varepsilon_{1}-\xi}} e^{i \phi_{1}}\right\}$.

Here, $\xi=\left(q / k_{0}\right), \phi_{1}=2 k_{0} z_{0} \sqrt{\varepsilon_{1}-\xi}$, and $r$ is the effective amplitude of reflection:

$r=\left[r_{s 12}+\left(\frac{\xi}{\varepsilon_{1}}-1\right) r_{p 12}\right] \sin ^{2} \vartheta+\frac{2 \xi}{\varepsilon_{1}} r_{p 12} \cos ^{2} \vartheta$, 
where $\vartheta$ is the angle between the vector $\mathbf{d}_{21}$ and the interface normal. Note that the relative spontaneous emission rate determined by Eqs (33), (34) coincides with that given by the classical theory [13]. Our calculations show that such agreement takes place also for an atom placed near a multilayer absorbing structures and semi-transparent slab. The obtained agreement between the classical and quantum theories (in the dipole approximation) forms a check on the validity of our quantization scheme.

We now consider the spontaneous emission for intersubband transitions in a QW. The infinite deep rectangular $\mathrm{QW}$ of the dimension $L_{w}$ is placed in the non-absorbing dielectric 1 parallel to the absorbing cladding layer $(0 \leq z \leq l)$. The $\mathrm{QW}$ center is at the distance $z_{0}$ from the interface $z=0$. We consider the direct transitions from the first excited state to the ground state (parabolic electron subbands). Note that only photons with $p$ polarization provide the transitions. Our estimations show that $\left|k_{1 z}\right| L_{w}<<1$, so that one may use the dipole approximation. The bulk rate of the spontaneous emission is given by Eq. (32) where now

$\mathbf{d}_{21}=\left(0,0, d_{21}\right), d_{21}=\left(\frac{4}{3 \pi}\right)^{2} e L_{w}, \omega=\frac{3 \pi^{2} \hbar}{2 m L_{w}^{2}}$,

$m$ is the electron effective mass. For the relative emission rate, one gets Eq. (33) where $r$ is determined by Eq. (34) with $\vartheta=0$.

We will study two types of absorbing media that can support the surface-phonon polaritons (polar dielectric) and the surface-plasmon polaritons (doped semiconductor or metal). For polar dielectric, the dielectric permittivity is described by an oscillator model:

$\varepsilon(\omega)=\varepsilon_{\infty} \frac{\omega_{l}^{2}-\omega^{2}-i \Gamma \omega}{\omega_{t}^{2}-\omega^{2}-i \Gamma \omega}$,

where $\varepsilon_{\infty}$ is the permittivity for high frequencies, $\omega_{l}$ and $\omega_{t}$ are the frequencies of longitudinal and transverse optical phonons, and $\Gamma$ is the damping. For a medium with a free electron gas, the dielectric permittivity is given by the Drude model:

$\varepsilon(\omega)=\varepsilon_{\infty}-\frac{\omega_{p}^{2}}{\omega(\omega+i / \tau)}$,

where $\omega_{p}$ is the plasma frequency, and $\tau$ is the plasmon relaxation time.

Fig. 1 provides the related emission rate as a function of the wavelength of emitted light $\lambda=2 \pi c / \omega$ for the QW placed near the $\operatorname{SiC}$ layer $\left(z_{0}<<\lambda\right)$. The different $\lambda$ can correspond to the QWs with different thicknesses $\left(\lambda \propto L_{w}{ }^{2}\right)$. The transition wavelength can also be changed by an applied electric field. For numerical evaluation, the GaAs quantum well was chosen $\left(\varepsilon_{1}=11, m=0.067 m_{0}\right.$, where $m_{0}$ is the free electron mass) and the following parameters of $\mathrm{SiC}$ [14] were used: $\quad \varepsilon_{\infty}=6.7, \quad \omega_{t}=149.5 \times 10^{12} \mathrm{~s}^{-1}$ $\left(\lambda_{t}=12.6 \mu \mathrm{m}\right), \quad \omega_{l}=182.7 \times 10^{12} \mathrm{~s}^{-1} \quad\left(\lambda_{l}=10.3 \mu \mathrm{m}\right)$, and $\Gamma=0.9 \times 10^{12} \mathrm{~s}^{-1}$. The curves 1 to 3 show how the thickness $l$ of SiC layer affects the emission rate. Note that emission of the photon modes $\{\omega, \mathbf{q}, p, e m\}$ provides the leading contribution to the rate. The enhancement of the radiative decay in a narrow spectral interval is caused by emission of the evanescent waves with the large wave vector $\mathbf{q}$. The wave vector becomes very large for a wavelength such that $\operatorname{Re} \varepsilon\left(\lambda_{\text {res }}\right)+\varepsilon_{1}=0$. The solution of this equation $\left(\lambda_{\text {res }}=11.57 \mu \mathrm{m}\right)$ determines the surface-polariton resonance in the semi-infinite $\mathrm{SiC}$. For $l \geq 0.5 \mu \mathrm{m}$, the $\mathrm{SiC}$ layer may be considered as a semi-infinite sample, and the position of the maximal magnitude of the emission rate $\tau^{-1}(\lambda)$ is approximately equal $\lambda_{\text {res }}$ (curves 1,2 , and 4 ). For curve $1, \lambda_{\max } \approx 11.6 \mu \mathrm{m}$ corresponds to the QW thickness $L_{w} \approx 125 \AA$. As the thickness $l$ of $\mathrm{SiC}$ layer is reduced, the dispersion relation for polariton starts to depend upon $l$, and $\lambda_{\max }$ increases with a decreasing of $l$ (curves 2 and 3 ). Comparing curves 1 and 4, we see that for QW placed in the near field of the polar layer $\left(z_{0}<<\lambda\right)$ the emission rate fast decreases when the distance of QW to the layer increases. From Eqs (33) and (34) for small distances $\left(z_{0} k_{0}<<1\right)$ and large in-plane wave vectors $\left(q^{2}>>\varepsilon_{1} k_{0}{ }^{2}\right)$, we get the dependence $\tau_{0} / \tau \propto 1 / z_{0}{ }^{3}$. Note that the $1 / z_{0}{ }^{3}$ dependence of the spontaneous rate corresponds to the variation of the electromagnetic energy density with the distance from the surface of absorbing medium [15].

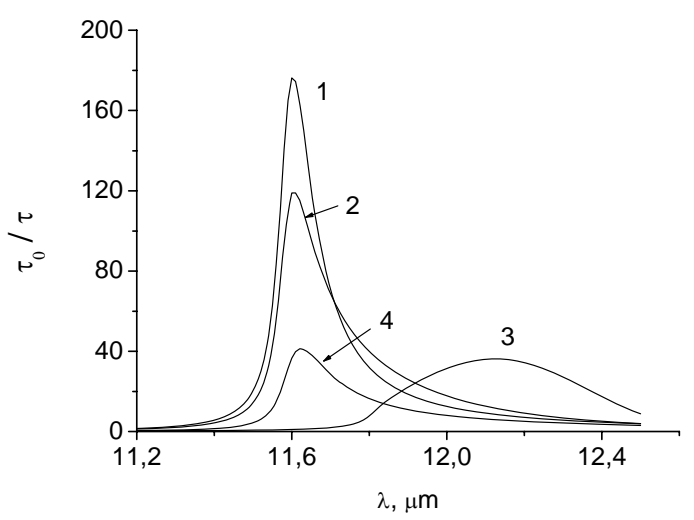

Fig. 1. Relative rate $\tau_{0} / \tau$ of spontaneous photon emission from QW versus the wavelength of the emitted light for different thicknesses $l$ of SiC layer and distances $z_{0}$ between QW and the interface. For $z_{0}=0.2 \mu \mathrm{m}, l=1 \mu \mathrm{m}(1), 0.25 \mu \mathrm{m}$ (2), and $0.05 \mu \mathrm{m}$ (3); for $z_{0}=0.3 \mu \mathrm{m}, l=1 \mu \mathrm{m}$ (4). 


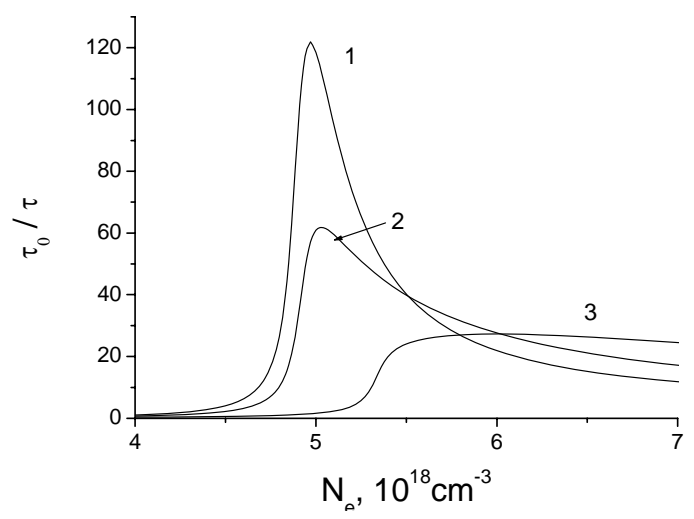

Fig. 2. Relative rate $\tau_{0} / \tau$ of spontaneous photon emission from QW placed at the distance $z_{0}=0.3 \mu \mathrm{m}$ from a layer of InAs with the thickness $l$ versus the electron concentration $N_{e}$ in the layer: $l=1 \mu \mathrm{m}(1), 0.3 \mu \mathrm{m}(2)$, and $0.15 \mu \mathrm{m}(3)$.

The related spontaneous emission rate for intersubband transitions in the QW placed near the InAs layer is presented in Fig. 2 as a function of the electron concentration $N_{e}$ in the layer. The calculations were carried out for the GaAs quantum well with the thickness $L_{w} \approx 140 \AA$ (the wavelength of emitted light equals $14.6 \mu \mathrm{m})$. For InAs, the following parameters were used: $\varepsilon_{\infty}=12.3, \quad m^{*}=0.04 m_{0}, \quad \tau=8 \times 10^{-13} \mathrm{~s}$. Fig. 2 explores the variation of the emission rate with the thickness $l$ of the absorbing layer, the distance of the QW from the nearest interface $z_{0}=0.3 \mu \mathrm{m}$. If $l \geq 1 \mu \mathrm{m}$, then the InAs layer can be treated as a semi-infinite medium, and the wavelength $\lambda_{\text {res }}$ corresponding to surface plasmon resonance is determined by the equation $\operatorname{Re} \varepsilon\left(\lambda_{\text {res }}\right)+\varepsilon_{1}=0$. Taking into account that the peak (curve 1) corresponds to the concentration $N_{e}=5 \times 10^{18} \mathrm{~cm}^{-3}$, we find $\lambda_{\text {res }}=14.4 \mu \mathrm{m}$. For $l<1 \mu \mathrm{m}$, the far side of the layer affects the emission rate (curves 2 and 3 ). We see that the spontaneous emission rate can be controlled not only by the variation of the electron concentration (due to doping or injection) but by changing the thickness of the absorbing top layer as well.

\section{Conclusion}

We have presented formalism for the electromagnetic field quantization in the presence of a planar multilayer absorbing structure. Field quantization was carried out by computing the complete set of orthonormal modes that are the solutions of the Maxwell equations for the macroscopic electromagnetic field with the appropriate boundary conditions. We have used the following two types of the solutions. One type defines the electromagnetic field related to plane waves that are incident from the non-absorbing dielectric half space for both orthogonal polarizations. Another type is the field generated by the current fluctuations in the absorbing medium. The modes are normalized with respect to the electromagnetic energy flow. It is assumed that the averaged over time normal to the surface component of the Poynting vector, which is associated with the incident wave of the frequency $\omega$, equals to the density of energy flow of the quanta $\hbar \omega$. This condition determines the amplitudes of the incident waves. To determine the amplitudes of the waves emitted by the absorbing medium, we assume that the total electromagnetic energy flow for the given frequency, direction, and polarization is absent.

To demonstrate the applicability of the theory, we have calculated the rate of the spontaneous photon emission of an excited two-level atom located in a nonabsorbing dielectric near an absorbing and dispersing medium. We have proved that the relative spontaneous emission rate determined in the dipole approximation coincides with that given by the classical point-dipole theory. We have also calculated the rate of photon emission for transitions between electron subbands in a quantum well placed nearby an absorbing layer supporting surface phonon or plasmon polaritons. When the separation between the quantum well and layer is much smaller than the radiation wavelength (the nearfield zone) the rate was found to be resonant increased.

\section{References}

1. N.A. Vlasenko, S.I. Pekar, and V.S. Pekar, Theory of spontaneous and stimulated chemiluminescence of ZnS-Mn layers // Zhurnal eksperiment. teor. fiziki 64, p. 371 (1973), in Russian.

2. H.P. Urbach and G.L.J.A. Rikken, Spontaneous emission from a dielectric slab // Phys. Rev. A 57, p. 3913 (1998).

3. S.-T. $\mathrm{Wu}$ and C. Eberlein, Quantum electrodynamics of an atom in front of a nondispersive dielectric half-space // Proc. Roy. Soc. London A 455, p. 2487 (1999).

4. V.I. Pipa, V.V. Mitin, and M. Stroscio, Photons in semibounded dielectric and the surface effect on spontaneous emission in nanostructures // Phys. Rev. B 65, 235308 (2002).

5. V.S. Pekar, Theory of spontaneous and stimulated emission of electromagnetic waves in unidimensionally inhomogeneous media and resonators // Zhurnal eksperiment. teor. fiziki 67, p. 471 (1974), in Russian.

6. M. Janowicz, D. Reddig, and M. Holthaus, Quantum approach to electromagnetic energy transfer between two dielectric bodies // Phys. Rev. $A$ 68, 043823 (2003).

7. R. Matloob, R. Loudon, S.M. Barnett, and J. Jeffers, Electromagnetic field quantization in absorbing dielectrics // Phys. Rev. A 52, p. 4823 (1995). 
8. R. Matloob, R. Loudon, Electromagnetic field quantization in absorbing dielectrics. II // Phys. Rev. A 53, p. 4567 (1996).

9. T. Gruner and D.-G. Welsch, Green-function approach to the radiation-field quantization for homogeneous and inhomogeneous Kramers-Kronig dielectrics // Phys. Rev. A 53, p. 1818 (1996).

10. S. Scheel, L. Knol, and D.-G. Welsch, QED commutation relations for inhomogeneous Kramers-Kronig dielectrics // Phys. Rev. A, 58, p. 700 (1998).

11. H.T. Dung, L. Knol, and D.-G. Welsch, Spontaneous decay in the presence of dispersing and absorbing bodies: General theory and application to a spherical cavity // Phys. Rev. A, 62, 053804 (2000).
12. J.E. Sipe, New Green-function formalism for surface optics // J. Opt. Soc. Amer. B 4, p. 481 (1987).

13. E.A. Vinogradov, I.A. Dorofeev, Thermostimulated electromagnetic fields of solid states // Uspekhi fiz. nauk, 179, p. 449 (2009), in Russian.

14. A.V. Shchegrov, K. Joulain, R. Carminati, and J.J. Greffet, Near-field spectral effects due to electromagnetic surface excitations // Phys. Rev. Lett. 85, p. 1548 (2000).

15. S.M. Rytov, Y.A. Kravtsov, and V.I. Tatarskii, Principles of Statistical Radiophysics, Vol. 3: Elements of Random Fields. Springer-Verlag, Berlin, 1989. 